\title{
Anti-LRP/LR-Specific Antibody IgG1-iS18 Significantly Impedes Adhesion and Invasion in Early- and Late-Stage Colorectal Carcinoma Cells
}

\author{
Leila Vania, Carryn J Chetty, Eloise Ferreira, and Stefan FT Weiss
}

School of Molecular and Cell Biology, University of the Witwatersrand, Johannesburg, Republic of South Africa

\begin{abstract}
Cancer is a highly complex disease that has become one of the leading causes of death globally. Metastasis, a major cause of cancer deaths, requires two crucial events, adhesion and invasion. The 37kDa/67kDa laminin receptor (laminin receptor precursor/high-affinity laminin receptor (LRP/LR)) enhances these two steps, consequently aiding in cancer progression. In this study, the role of LRP/LR in adhesion and invasion of early-stage (SW-480 and HT-29) and late-stage (DLD-1) colorectal cancer cells was investigated. Western blotting revealed that early- and late-stage colorectal cancer cells contained significantly higher total LRP/LR levels compared with poorly invasive MCF-7 breast cancer control cells. Flow cytometry revealed that both stages of colorectal cancer displayed significantly higher cell surface LRP/LR levels. Furthermore, upon treatment of colorectal cancer cells with the anti-LRP/LR-specific antibody IgG1-iS18, adhesion to laminin-1 was significantly reduced in both stages. Each stage's invasive potential was determined using the Matrigel ${ }^{\mathrm{TM}}$ invasion assay, showing that invasion was significantly impeded in both colorectal cancer stages when the cells were incubated with IgG1-iS18. In addition, Pearson's correlation coefficients propose that both total and cell surface LRP/LR levels are directly proportional to the adhesive and invasive potential of both stages of colorectal cancer. Hence, these findings indicate potential for use of the IgG1-iS18 antibody as a promising therapeutic tool for colorectal cancer patients at both stages.
\end{abstract}

Online address: http://www.molmed.org

doi: 10.2119/molmed.2016.00169

\section{INTRODUCTION}

Cancer, a highly complex disease, has become one of the leading causes of death globally. The World Health Organization predicts a $75 \%$ increase in total cancer cases worldwide by the year 2030 (1). More relevantly, South Africa ranks 50th in highest cancer incidences, and a recent study suggests that South Africa could face a $78 \%$ increase in the number of cancer cases by 2030 (2).

The present study focuses on colorectal cancer, the third most common cancer type, with over 1.4 million new cases diagnosed in 2012, including 600,000 deaths (http:/ / www.wcrf.org/int/ cancer-facts-figures/worldwide-data). Untreated colorectal cancer is the second most fatal type after lung cancer, yet if diagnosed in its early stages, it can be effectively treated (2). Colorectal cancer can be classified into four primary stages: early (stage I), middle (stages II and III) and late (stage IV), which results in metastasis.

According to Hanahan and Weinberg (3), there are several well-known hallmarks of cancer. These include activation of growth pathways, suppression of growth-inhibiting pathways, inhibition

Address correspondence to Stefan FT Weiss, School of Molecular and Cell Biology, University of the Witwatersrand, Private Bag 3, Wits 2050, Johannesburg, Republic of South Africa.E-mail: stefan.weiss@wits.ac.za

Submitted July 20, 2016; Accepted for Publication September 6, 2016; Published Online (www.molmed.org) September 8, 2016.

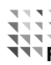

Feinstein Institute
for Medical Research

Northwell Health"

of apoptosis, enhancement of angiogenesis, and tissue invasion and metastasis, the latter being the focus of the present study. It has also been proven that cancerous cells are able to adhere to and invade secondary sites through the mediation of integrin and nonintegrin receptors (4). More specifically, the nonintegrin receptor $37 \mathrm{kDa}$ laminin receptor precursor/67kDa high-affinity laminin receptor (LRP/LR) has been shown to be notably overexpressed in various cancer types (4). This overexpression is found to have a direct correlation to the degree of adhesive and invasive potential of several cancer types (5).

$\mathrm{LRP} / \mathrm{LR}$ is a nonintegrin cell surface receptor located in the extracellular matrix of mammalian cells $(6,7)$. While LRP/LR predominantly functions as a transmembrane receptor (8), it has also been found in the nucleus, where it interacts with histones and chromatin (9), as well as in the cytosol, where it aids in translation and ribosomal 
biogenesis (10). Its physiological functions include cellular growth, adhesion, invasion, movement and viability (10). LRP/LR has been found to be a major contributor to the pathogenesis of neoplastic cancers (10), angiogenesis enhancement (12), prion disorders (13-15) and neurodegenerative diseases such as Alzheimer's disease (16-20). In addition, upregulation of the receptor has been seen to be implicated in telomerase activity (21). Research has shown that LRPmRNA encodes for the $37 \mathrm{kDa}$ laminin receptor precursor, which is the precursor protein for the $67 \mathrm{kDa}$ high-affinity laminin receptor (22). However, the exact mechanism by which the $67 \mathrm{kDa} \mathrm{LR}$ is formed is not known.

When LRP/LR is located on the cell surface, it is known to aid in organization of the basement membrane (22). Furthermore, it has been found that LRP/LR exhibits a high affinity for laminin-1, an essential element of the basement membrane (22). Laminin-1 is a noncollagenous, heterotrimeric glycoprotein that is able to bind to the extracellular matrix (ECM) (23). Therefore, laminin-1 functions as a key player in enhancing biological processes such as cell adhesion, homing (24), polarity, migration, proliferation, differentiation (25) and neutrite outgrowth (26). Research has shown that laminin-1 also enhances the invasive phenotype of cancerous cells, promoting tumor metastasis (27). Overexpression of LRP/LR on tumorigenic cell surfaces is a result of its increased interaction with laminin-1 (5). This increased interaction subsequently leads to increased adhesion, followed by activation of Type IV collagenase, leading to degradation of the basement membrane and invasion by the tumorigenic cells (28). Overexpression of LRP/LR, when compared with noninvasive tumorigenic cells, has been observed in various invasive tumors, including pancreatic cancer cells (29); colon, lung, prostate and cervical cancer cells (30); esophageal and breast cancer cells (31); and liver cancer cells (8).

Since the interaction between LRP/LR and laminin-1 is directly proportional to the degree of metastasis, inhibiting this interaction may serve as a crucial mechanism in treating the progression of metastatic cancer. Additionally, it has been found that IgG1-iS18, the full-length anti-LRP/LR-specific antibody, exhibits a significant effect on hampering the LRP/LR-laminin-1 interaction in various cancers, including early-stage colorectal cancer (30).

Thus, by applying the anti-LRP/LRspecific antibody IgG1-iS18 to early- and late-stage colorectal cancer cells, the stage where the antibody is most effective can be determined. Due to the rise in prevalence and mortality rate of colorectal cancer, it is crucial to develop a novel treatment strategy. Hence, the present study aimed to investigate the capacity of IgG1-iS18 to hamper the adhesive and invasive potential in both stages of colorectal cancer, thus allowing IgG1-iS18 to serve as a promising therapeutic tool in the treatment of colorectal cancer.

\section{MATERIALS AND METHODS}

\section{Cell Culture and Conditions}

Early-stage human colorectal adenocarcinoma (SW-480), late-stage human colorectal adenocarcinoma (DLD-1) and human poorly invasive adenocarcinoma (MCF-7) cell lines were obtained from the American Tissue Culture Collection. Early-stage human colorectal adenocarcinoma (HT-29) was obtained from Fox Chase Cancer Centre. All three colorectal cancer cell lines were cultured in Dulbecco's Modified Eagle Medium (DMEM) / Hams F12 (1:1), while the MCF-7 cells were cultured using DMEM high glucose (4.5 g/L). Each medium was supplemented with $10 \%$ fetal calf serum and $1 \%$ penicillin/streptomycin, and the cells were incubated at $37^{\circ} \mathrm{C}$ in a $5 \% \mathrm{CO}_{2}$ humidified atmosphere.

\section{Reagents and Antibodies}

Matrigel $^{\mathrm{TM}}$ used for cell invasion assays was derived from the EngelbrethHolm-Swarm (EHS) mouse sarcoma and was obtained from Corning Inc.
Laminin-1 used for adhesion assays was obtained from Sigma-Aldrich. Monoclonal antibody IgG1-HD37 (Sigma-Aldrich) possessing the same format as primary antibody IgG1-iS18 is directed against CD19 on $\beta$-lymphocytes in humans. The full-length antibody IgG1-iS18 was recombinantly produced in a mammalian expression system as previously described by Zuber et al. (6).

\section{Confocal Microscopy}

Confocal microscopy was used for visualization and confirmation of LRP / LR on the cell surface using fluorescentlabeled antibodies. Cells were first fixed in $4 \%$ paraformaldehyde (PFA) in $1 \times$ phosphate based saline (PBS) followed by numerous washes with PBS (Gibco). Thereafter, the cells were blocked using $0.5 \%$ bovine serum albumin (BSA) in PBS and washed again in PBS wash, and the excess PBS was blotted off. Thereafter, antibody diluted in PBS and 0.5\% BSA was added. The slides containing the coverslips were then incubated overnight at $4^{\circ} \mathrm{C}$. Following incubation, three PBS/ BSA washes were performed, after which fluorescein isothiocynate (FITC)-coupled secondary antibody (Abcam), which was diluted in PBS and 0.5\% BSA, was added. This was followed by $1 \mathrm{~h}$ incubation in the dark. Thereafter, three more PBS/BSA washes took place, followed by the addition of Hoescht stain (SigmaAldrich). This was followed by a 5-10 min incubation and then rinsing in PBS. GelMount (Sigma-Aldrich) was used for mounting onto a new slide.

\section{Fluorescence-Activated Cell Sorting}

For evaluation and quantification of cell surface LRP/LR levels, flow cytometry was used. Cells were first detached using $1 \times$ Trypsin/Versene, centrifuged at $150 \times g$ for $10 \mathrm{~min}$ and resuspended and fixed with $1 \mathrm{~mL}$ of $4 \%$ PFA for 10 min. Thereafter, three cell suspensions were prepared by resuspending in 100 $\mu \mathrm{L}$ of PBS. One cell suspension was incubated with the primary antibody IgG1-iS18 $(30 \mu \mathrm{g} / \mathrm{mL})$, the second was incubated with the control antibody 
IgG1-HD37 $(30 \mu \mathrm{g} / \mathrm{mL})$ and the third was resuspended in $1 \times$ PBS only, serving as the negative control. This was followed by incubation overnight at $4^{\circ} \mathrm{C}$. Thereafter, the cell pellets were washed three times in $300 \mu$ of PBS through centrifugation at $2700 \times g$ for $5 \mathrm{~min}$. Following this, all cell pellets were resuspended in PBS and goat antihuman phycoerythrin (PE)-coupled secondary antibody (1:1000) (Abcam), before incubating both cell suspensions for $1 \mathrm{~h}$ in the dark. The cell suspensions were then washed three more times in PBS and evaluated using the BD Accuri C6 flow cytometer and software. Three biological replicates of the experiment were performed with different cell cultures.

\section{SDS-PAGE and Western Blotting}

Western blotting was used to detect total LRP/LR levels and compare LRP/ LR levels at each stage of colorectal cancer. An amount of $11 \mu \mathrm{g}$ of total protein was subjected to sodium dodecyl sulphatepolyacrylamide gel electrophoresis (SDSPAGE). The resulting separated proteins were transferred onto a polyvinylidine fluoride membrane (Pall) with $1 \times$ transfer buffer (20\% methanol in $25 \mathrm{mM}$ Tris and $19.2 \mathrm{mM}$ glycine) at $450 \mathrm{mV}$ for $45 \mathrm{~min}$. The membrane was then blocked with blocking buffer $(1 \times$ PBS-Tween [0.1\% Tween in 1XPBS] with $3 \%$ BSA) for $1 \mathrm{~h}$ to prevent nonspecific binding of primary and secondary antibodies. The membrane was then probed with the primary anti-LRP/LR-specific antibody IgG1-iS18 (1:10000) for $1 \mathrm{~h}$. Thereafter, the membrane was washed three times with $1 \times$ PBS-Tween before incubating with goat antihuman horseradish peroxidase (HRP) secondary antibody (1:5000) (Abcam). As a loading control, $42-\mathrm{kDa} \beta$-actin was used, and was detected by means of the monoclonal anti- $\beta$-actin-peroxidase. Every experiment was performed in triplicate with different cell cultures.

\section{Adhesion Assay}

To evaluate the potential of colorectal cancer cell lines to adhere to the basement membrane, an in vitro adhesion assay was performed in 96-well plates coated with laminin-1 (10 $\mu \mathrm{g} / \mathrm{mL})$, with uncoated wells as negative controls. After coating the wells with laminin- 1 for $1 \mathrm{~h}$, the 96well plate was incubated with $100 \mu \mathrm{l}$ blocking solution containing $0.5 \%$ BSA in DMEM for $1 \mathrm{~h}$. Thereafter, cells were suspended in serum-free culture media and treated with $\operatorname{IgG} 1-\mathrm{iS} 18(0.2 \mathrm{mg} / \mathrm{mL})$, along with IgG1-HD37 (0.2 mg/mL) antibodies serving as negative control, to analyze the adhesive potential. The cells were then added to wells at a cell density of $4 \times 10^{5}$ cells $/ \mathrm{mL}$ and incubated for $1 \mathrm{~h}$. Following incubation, cells that did not attach were washed and removed with PBS, while attached cells were fixed in $50 \mu \mathrm{L}$ of $4 \%$ PFA for $10 \mathrm{~min}$, prior to staining with $100 \mu \mathrm{L}$ of $0.1 \%$ crystal violet for $10 \mathrm{~min}$. The dye was then extracted with $100 \mu \mathrm{L}$ of $2 \%$ SDS and the absorbance was measured at $550 \mathrm{~nm}$ using an ELISA plate reader. This absorbance was an indicator of cells that were attached to laminin-1. Experiments were performed in triplicate with different cell cultures.

\section{Invasion Assay}

To evaluate the potential of the colorectal cancer cell lines to invade the basement membrane, an in vitro invasion assay was performed. Matrigel ${ }^{\mathrm{TM}}$ was diluted with cold serum-free cell culture medium (Thermo Scientific) and placed onto the upper chamber of a 24well transwell plate (BD Falcon, $8 \mu \mathrm{m}$ pore size). The gel was then incubated at $37^{\circ} \mathrm{C}$ for $4 \mathrm{~h}$, until it was solidified. The cells were harvested and washed with culture medium containing $1 \%$ fetal calf serum at a cell density of $1 \times$ $10^{6}$ cells $/ \mathrm{mL}$. Following this, the cells were incubated with the IgG1-iS18 $(0.2 \mathrm{mg} / \mathrm{mL})$ antibody or the control IgG1-HD37 (0.2 mg/mL) antibody for $10 \mathrm{~min}$. The cells were then loaded onto the transwell inserts, and the lower chamber was filled with $500 \mu$ l serumfree culture medium for the control and $500 \mu \mathrm{l}$ culture medium containing 10\% fetal calf serum for the experimental cell lines. After incubation at $37^{\circ} \mathrm{C}$ for $18 \mathrm{~h}$, both the upper and lower media were removed. The noninvasive cells were then removed with a cotton swab and the invasive cells were fixed with $100 \mu \mathrm{L}$ of $4 \%$ PFA and rinsed with $100 \mu \mathrm{L}$ of PBS, followed by staining with $30 \mu \mathrm{L}$ of $0.5 \%$ Toluidine blue for $2 \mathrm{~min}$. The dye was then extracted with $100 \mu \mathrm{l}$ of $1 \%$ SDS for $30-45 \mathrm{~min}$. The absorbance of the resultant solutions was then obtained at $620 \mathrm{~nm}$ using an ELISA plate reader. Three biological repeats were performed.

\section{Statistical Analysis}

To analyze the data accurately, the two-tailed Student $t$ test with a confidence interval of $95 \%$ was used, with $p$ values greater than 0.05 considered not significant ${ }^{*} p<0.05,{ }^{* *} p<0.01$ and *** $p<0.001)$. Error bars represent standard deviation. To measure the degree of association between LRP/LR levels and adhesive/invasive potential, Pearson's correlation coefficient was calculated. A positive coefficient shows direct proportionality between the two variables, while a negative coefficient implies inverse proportionality.

All supplementary materials are available online at www.molmed.org.

\section{RESULTS}

\section{Early- and Late-Stage Colorectal Cancer Cells Exhibit LRP/LR on the Cell Surface}

Research has shown that the interaction between laminin-1 and the $37 \mathrm{kDa} / 67 \mathrm{kDa}$ laminin receptor precursor (LRP/LR) is essential for metastasis to occur $(24,25)$. Thus, visualization of LRP/LR on the cell surface was needed to confirm that these tumorigenic cells exhibited LRP/LR on their cell surfaces. In Figure 1, it can be seen that all three colorectal cancer cell lines, as well as the poorly invasive breast cancer (MCF-7) cells posing as control, contained LRP/ LR on the cell surface. All cells were nonpermeabilized, thus the green fluorescence depicted below indicates cell surface LRP/LR. 
A IgG1-iS18

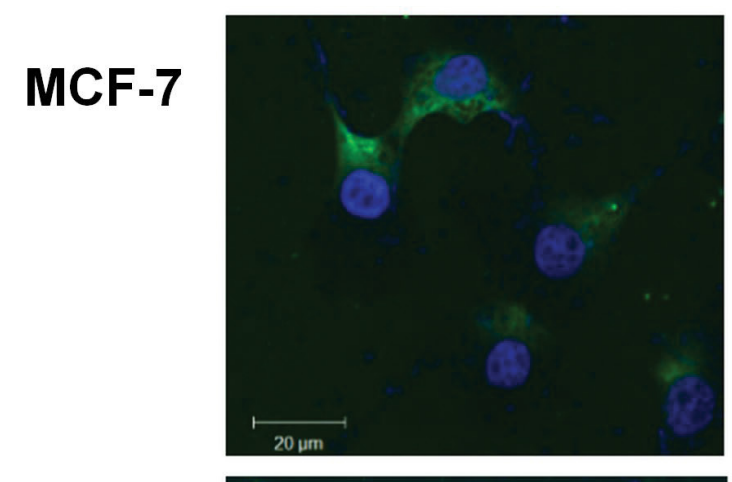

\section{SW-480}

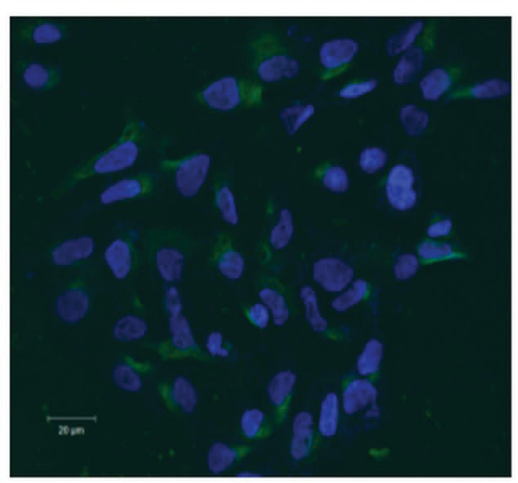

HT-29

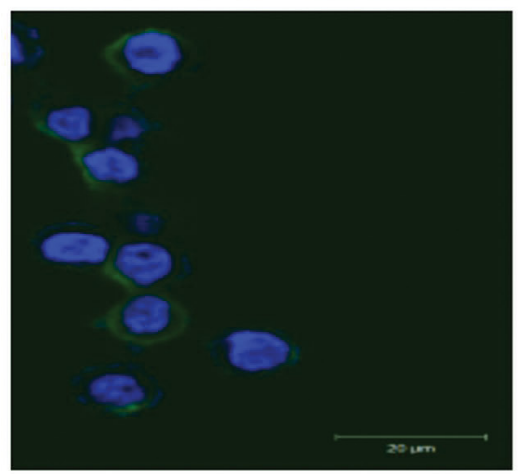

\section{DLD-1}

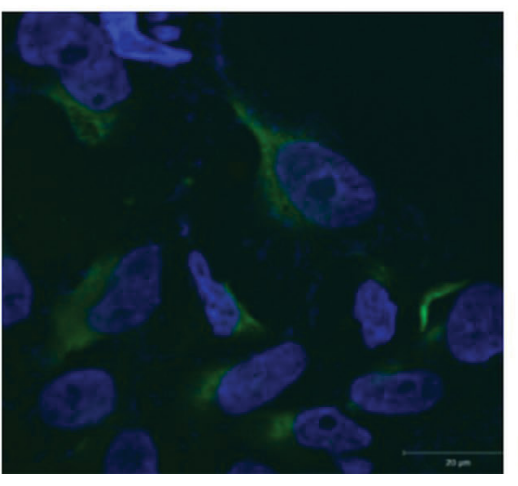

\section{NO IgG1-iS18}
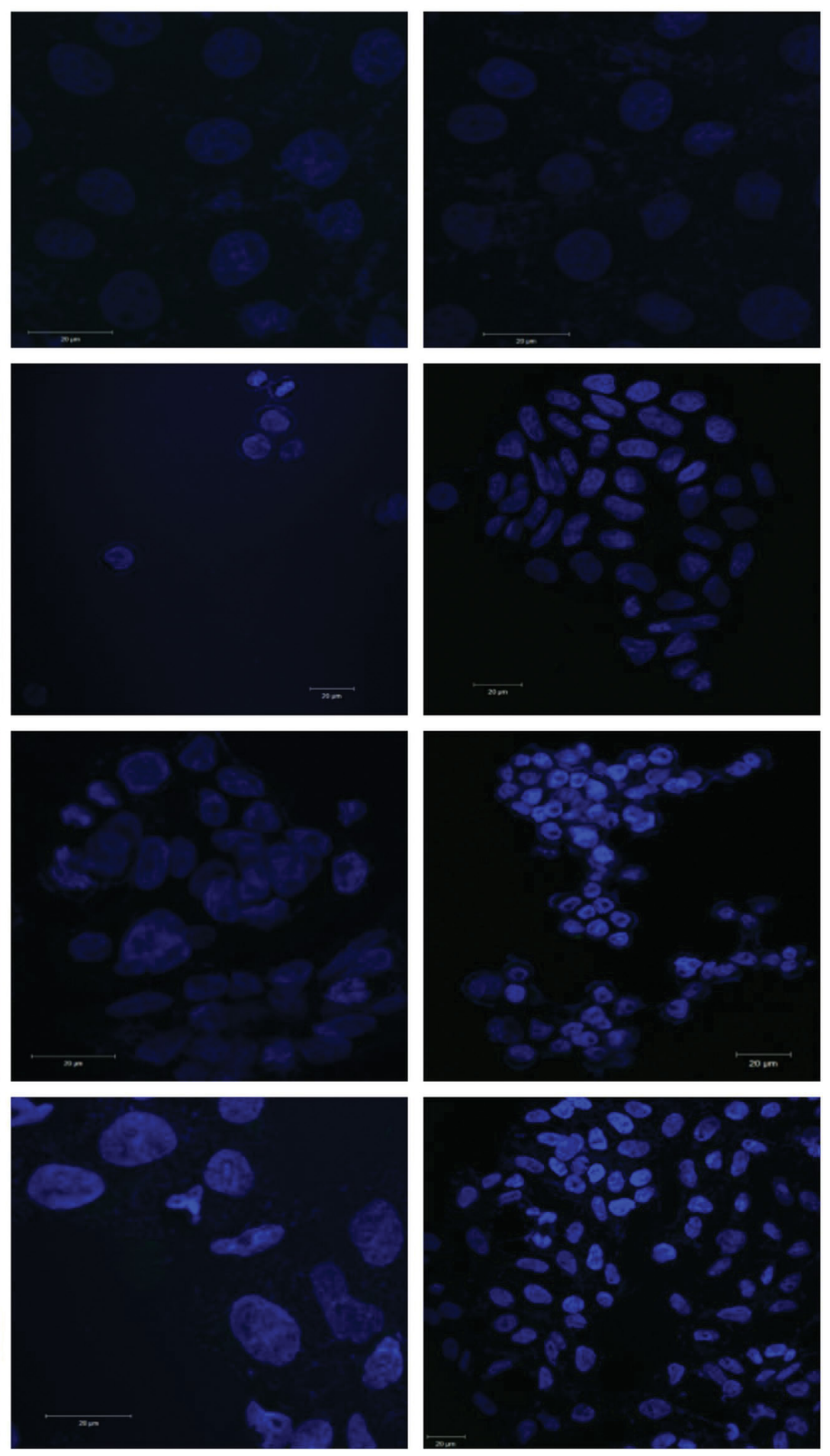

Figure 1. Visualization of LRP/LR on the surface of early-stage (SW-480 and HT-29) and late-stage (DLD-1) colorectal cancer and poorly invasive (MCF-7) tumorigenic cells. (A) Cells labeled with primary antibody lgG1-iS18 and FITC-coupled secondary antibody specific for lgG1-iS18 and found to fluoresce green. (B) Cells labeled with lgG1-HD37 antibody serving as negative control. (C) Cells labeled with only the FITC-coupled secondary antibody to confirm that there was no nonspecific binding coming from the secondary antibody. 
Early- and Late-Stage Colorectal Cancer Cells Exhibit High Percentages Of LRP/LR and Significantly Greater LRP/LR Levels on the Cell Surface Compared to Poorly Invasive MCF-7 Controls

Despite confocal microscopy data confirming that LRP/LR is present on the cell surface of colorectal cancer cells at each stage, additional quantification of LRP/LR's being expressed was needed. This was done by flow cytometric analysis. All tumorigenic cell lines were treated alike to enable comparison of cell surface LRP/LR levels. In Figure 2, it can be seen that all three colorectal cancer cell lines, as well as the MCF-7 control cell line, exhibit high percentages of cells that possess LRP/LR on the cell surface within a particular population. This is indicated by the shift between the the black and blue peaks in each graph, depicting changes in fluorescent intensity, which is a result of staining the cell surfaces with anti-LRP/LRspecific antibody IgG1-iS18. As shown in Figure 2, SW-480, HT-29 and DLD-1 colorectal cancer cells display higher percentages $(97.69 \%, 91.25 \%$ and $89.28 \%$, respectively) of cells within a specific population that express LRP/LR on the cell surface, compared with the MCF-7 control cells (87.88\%), with SW-480

displaying the highest amount.

Once the percentage of cells expressing LRP/LR on the cell surface was analyzed, further quantification of the definite amount of cell surface LRP/LR within a particular population of cells was done with flow cytometry. Each tumorigenic cell line was treated alike; that is, the same amount of cells $(20,000)$ within specific populations were labeled with primary and secondary antibodies containing the same concentration (30 $\mu \mathrm{g} / \mathrm{mL}$ ) over the same duration. In Figure 3, it can be seen that both stages of colorectal cancer display significantly

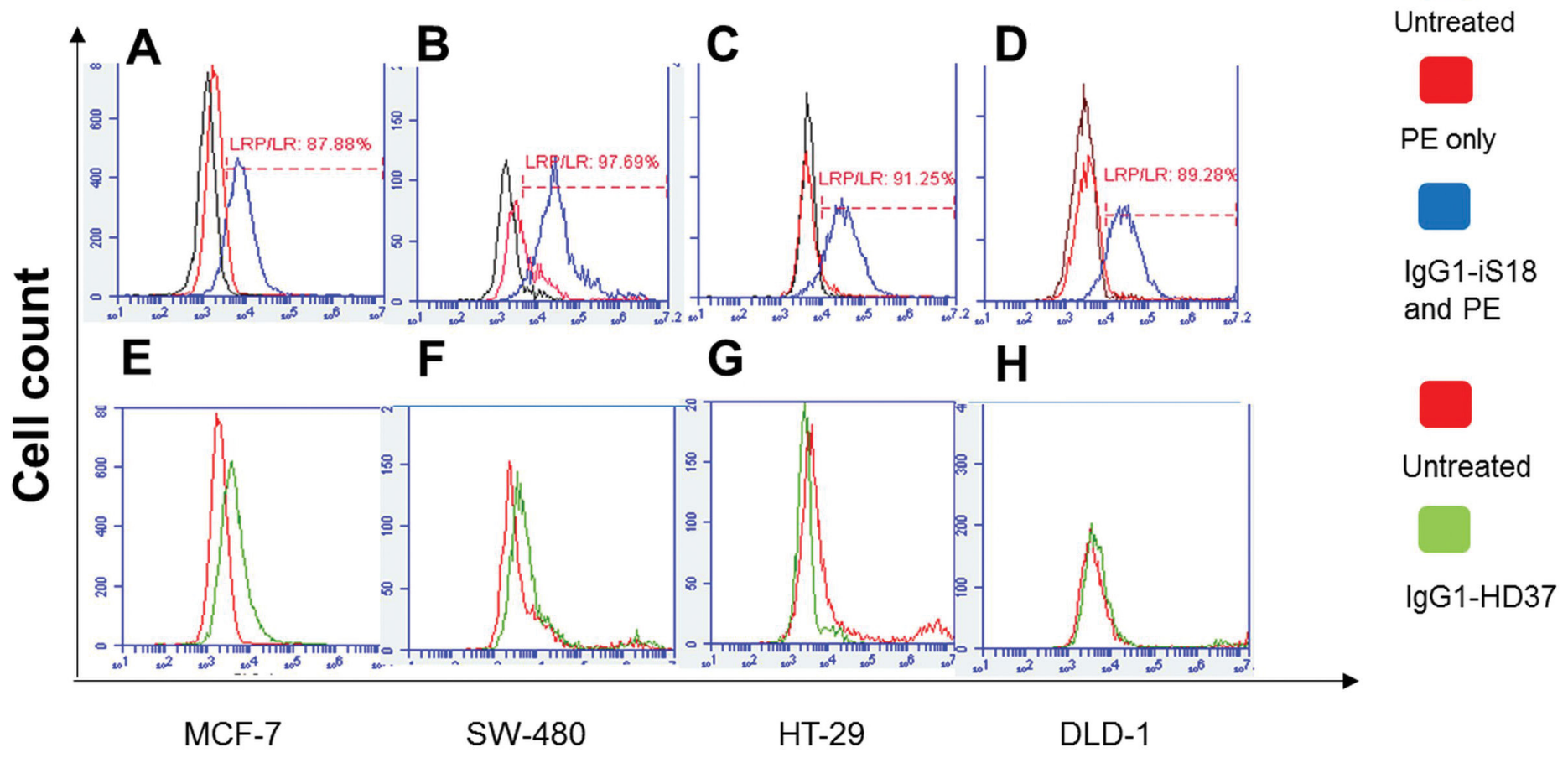

Fluorescence intensity (FL-2)

Figure 2. Detection of $37 \mathrm{kDa} / 67 \mathrm{kDa}$ LRP/LR levels on the surfaces of tumorigenic cell lines. Cells were treated with IgG1-iS18 and IgG1-HD37 (control antibody). (A and E) represent MCF-7, (B and F) represent SW-480, (C and G) represent HT-29, and (D and H) represent DLD-1. In graphs (A, B, C and D) the black peak represents untreated cells, the red peak indicates cells labeled with goat antihuman phycoerythrin-coupled secondary antibody only and the blue peak represents cells labeled with anti-LRP-specific antibody IgG1-iS18 together with secondary antibody, both at a concentration of $30 \mu \mathrm{g} / \mathrm{mL}$. In graphs $(E, F, G$ and $H)$ the red peak indicates untreated control to show no nonspecific binding from the secondary antibody; the green peak represents control antibody lgG $1-\mathrm{HD} 37$ together with the secondary antibody. These data represent experiments performed in triplicate using different cell cultures, with 20,000 cells counted per sample. 


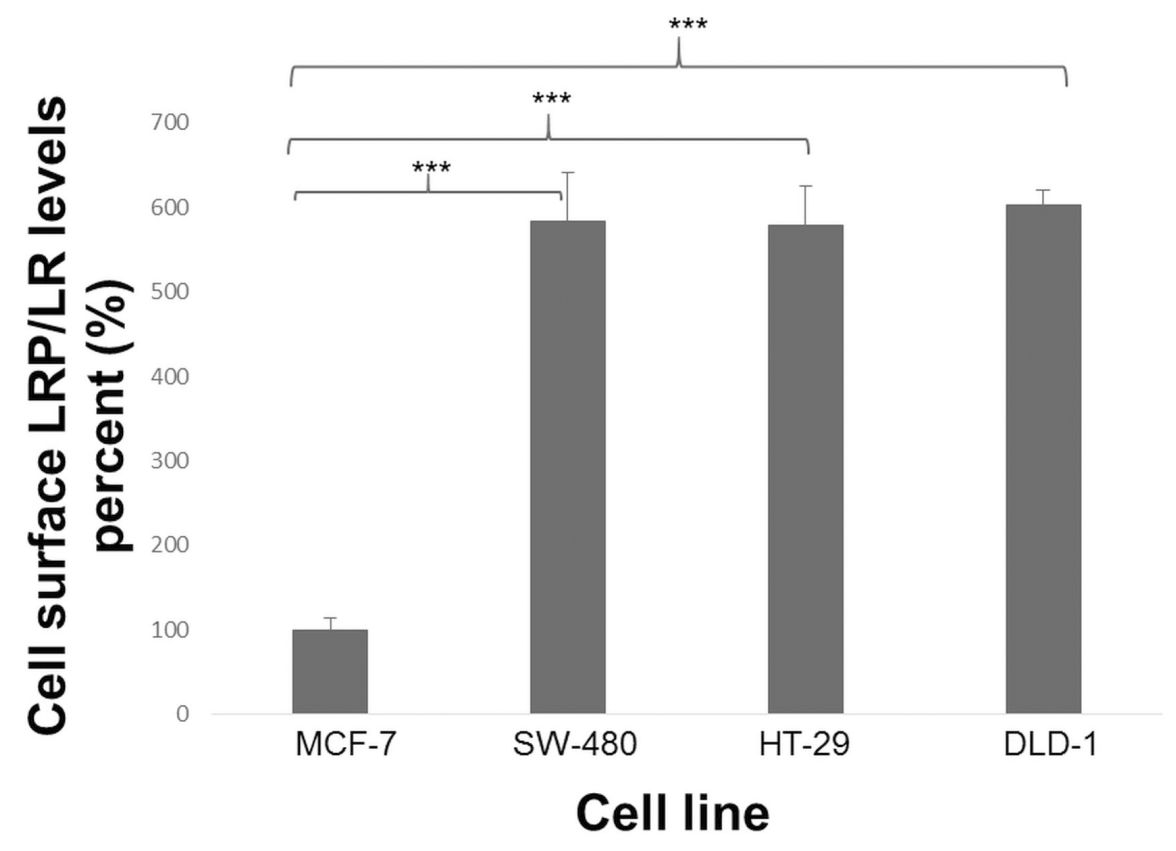

Figure 3. Quantification of cell surface LRP/LR levels on tumorigenic cell lines. Cells were labeled with lgG1-iS18 primary antibody and antihuman phycoerythrin-coupled secondary antibody. Thereafter, 20,000 cells from each tumorigenic cell line were analyzed and fluorescence intensities were used to indicate cell surface LRP/LR levels. These data represent experiments performed in triplicate using different cell cultures.

higher amounts of LRP/LR within a cell population compared with the MCF-7 cells. However, when comparing the colorectal cancer stages to one another, no significant difference was observed (see Supplementary Figure S1).

\section{Late-Stage Colorectal Cancer Cells Exhibit Significantly Higher Total LPR/ LR Levels Compared to MCF-7 Control Cells and Early-Stage Colorectal Cancer Cells}

Due to the fact that LRP/LR is expressed in the nucleus and cytosol as well as on the cell surface, Western blotting was performed to investigate total LRP/LR levels. It must be noted that only detection of the $37 \mathrm{kDa}$ LRP could be achieved through use of the anti-LRP / LR-specific antibody IgG1-iS18. As seen in Figure 4, all tumorigenic cell lines could be seen to express LRP/LR. In addition, late-stage colorectal cancer cells revealed a significant three-anda-half-fold increase in total LRP/LR levels compared with the MCF-7 cells.
Furthermore, there was a significant two-fold increase in total LRP/LR levels between the two early stages when compared with the late stage. There was no significant difference between the two early-stage colorectal cancer cell lines, as expected.

\section{IgG1-iS18 Significantly Impedes the Adhesive Potential of Early- and Late-Stage Colorectal Cancer Cells}

Before tumorigenic cells can invade, resulting in metastasis, adhesion to the basement membrane by means of the laminin-1-LRP/LR interaction has to occur. It is this interaction that causes other important cellular interactions, facilitating degradation of the basement membrane. Hence, each cell line's adhesive potential was evaluated through the use of laminin-1-coated plates. Each tumorigenic cell line was incubated with IgG1-iS18 and IgG1-HD37 antibodies; absorbance readings of the resulting solutions depicted the quantity of cells attached to the plates. In Figure 5, it can be seen that all three colorectal cancer cell lines showed more adhesive potential than the poorly invasive MCF-7 control cells. It can also be seen that application of IgG1-iS18 to the cells resulted in significant hampering of the adhesive potential of all three cell lines. However, no reduction in the poorly invasive MCF-7 control was seen. Late-stage colorectal cancer showed the highest adhesive potential as well as the highest decrease in adhesive potential.

\section{IgG1-iS18 Significantly Impedes Invasion of Matrigel ${ }^{\mathrm{TM}}$ by Early- and Late-Stage Colorectal Cancer Cells}

Before a tumorigenic cell line becomes metastatic, it must undergo a vital process, invasion through the basement membrane. Thus, invasion assays were performed to evaluate the invasive potential of each tumorigenic cell line using the Matrigel ${ }^{\mathrm{TM}}$ invasion assay. The gel is known to mimic the basement membrane, since it contains vital components of the ECM, including laminin-1. All cell lines were treated with IgG1-iS18 and IgG1-HD37. In Figure 6, it can be seen that all three colorectal cancer cell lines were significantly more invasive compared with the poorly invasive MCF-7, with late-stage being the most invasive. In addition, once IgG1-iS18 was applied, the invasive potential of all three colorectal cancer cell lines was successfully impeded.

\section{DISCUSSION}

Previous studies have proven that numerous cancerous cell lines display overexpressed levels of $37 \mathrm{kDa} / 67 \mathrm{kDa} \mathrm{LRP} / \mathrm{LR}$ (23-25). This suggests that the interaction between LRP/LR and laminin-1 may be essential for tumorigenic cells to transform into malignant cancers. Thus, by inhibiting this interaction, this may in turn inhibit two crucial events necessary for metastasis to occur, adhesion and invasion. Therefore, the purpose of this study was to investigate the role of LRP/LR in adhesion and invasion of early-stage (SW-480 and HT-29) and late-stage (DLD-1) colorectal cancer cells, and whether applying 
A
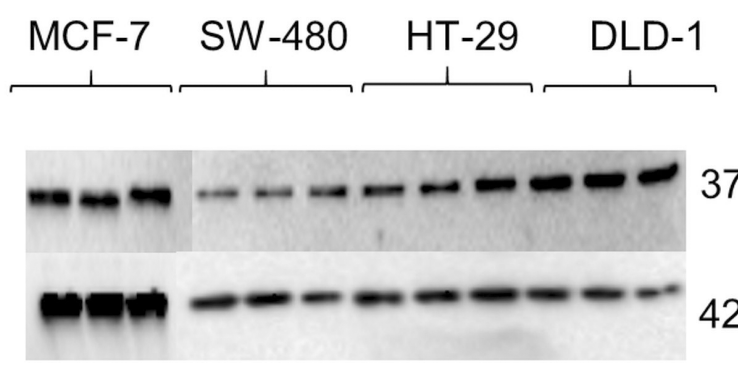

$37 \mathrm{kDa}$ LRP

$42 \mathrm{kDa} \beta$-actin

B

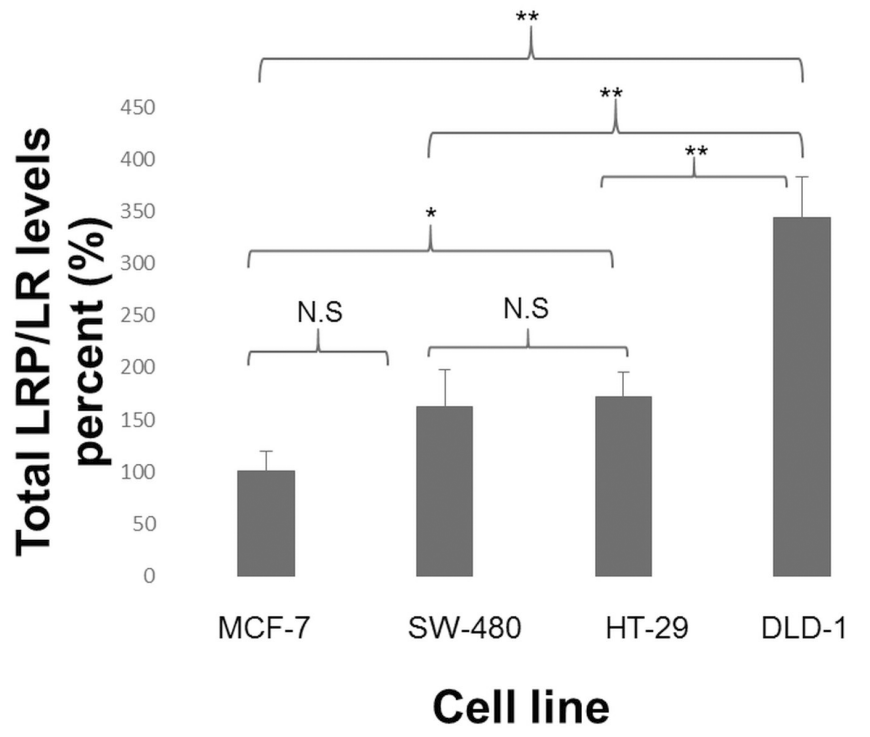

Figure 4. (A) Detection of total $37 \mathrm{kDa}$ LRP levels in protein lysates of MCF-7, SW-480, HT-29 and DLD-1 cells. IgG1-iS18 was used as the primary antibody, with HRP-coupled secondary antibody; $42 \mathrm{kDa} \beta$-actin was used as a loading control. (B) Densitometric analysis of total LRP/LR levels on SW-480, HT-29 and DLD-1 cell lines revealed that, compared to MCF-7, DLD-1 contained the most total LRP/LR, displaying a 3.5-fold increase. A significant two-fold increase in total LRP/LR levels between early- and late-stage colorectal cancer was also found. These data represent experiments performed in triplicate using different cell cultures.

IgG1-iS18, the anti-LRP/LR-specific antibody, would significantly impede their adhesive and invasive potential.

Confocal microscopy confirmed that all cell lines did display LRP/LR on their cell surfaces (Figure 1). However, this technique is only qualitative and does not reveal the levels of LRP/LR on the cell surface, thus further analysis using flow cytometry was performed.

Flow cytometry revealed that all three colorectal cancer cell lines displayed higher percentages of cells within a specific population that express cell surface LRP/LR compared with the MCF-7 control cells. Further analysis of each cell line's median fluorescence intensity revealed that all three displayed significantly higher amounts of LRP /

LR within a cell population, compared with the control MCF-7 cells (Table 1).

As mentioned above, LRP/LR is vital for processes such as cell adhesion, invasion, proliferation and migration, and therefore, since all three cell lines are known to be invasive (23), these high levels of cell surface LRP/LR may be contributing to the invasive potential of these cell lines.

Further analysis was done with Western blotting to evaluate total LRP/ LR levels, which includes LRP/LR found in the nucleus and cytosol of cancerous cell lines. Analysis of the Western blots (Figure 4) confirmed that each tumorigenic cell line expressed $37 \mathrm{kDa}$ LRP. Densitometric analysis of the blots (Figure 4B) revealed a significant three-and-a-half-fold increase in total LRP/LR levels in late-stage colorectal cancer, while both early stages showed no significant difference in the total levels when compared with the poorly invasive MCF-7 cells. It was also revealed that when the stages of colorectal cancer were compared with one another, there was a significant two-fold increase in total LRP/LR levels between early stage and late stage (Figure 4B). Besides contributing to the key steps of metastasis, it is important to note that LRP/LR also plays crucial roles in the nucleus and cytosol. This includes nuclear structure maintenance and translational processes (10). Hence, with increased levels of total LRP/ LR, cells acquire heightened levels of protein synthesis, which is necessary for processes that are involved in cancer progression and metastasis, such as tumor angiogenesis. As a result, the increased levels of total LRP/LR observed in DLD-1 compared with the other cell lines may contribute to its invasiveness, aiding in metastasis. The significant differences seen in cell surface and total LRP/LR levels reveal important roles in the progression of both stages of colorectal cancer, compared with the poorly invasive MCF-7 cells.

Analysis of the results obtained in this study suggests that IgG1-iS18 was able to cause a considerable decrease in the adhesive potential of all three colorectal cancer cell lines on laminin-1 as well as impede their invasive potential on Matrigel $^{\mathrm{TM}}$. These results suggest that differences in the adhesive and invasive potential of these cell lines may be a consequence of differences in total LRP / LR levels as opposed to cell surface LRP/LR levels. Although there are significant differences in cell surface LRP/ LR of these cell lines when compared with the MCF-7 cells, which are poorly 


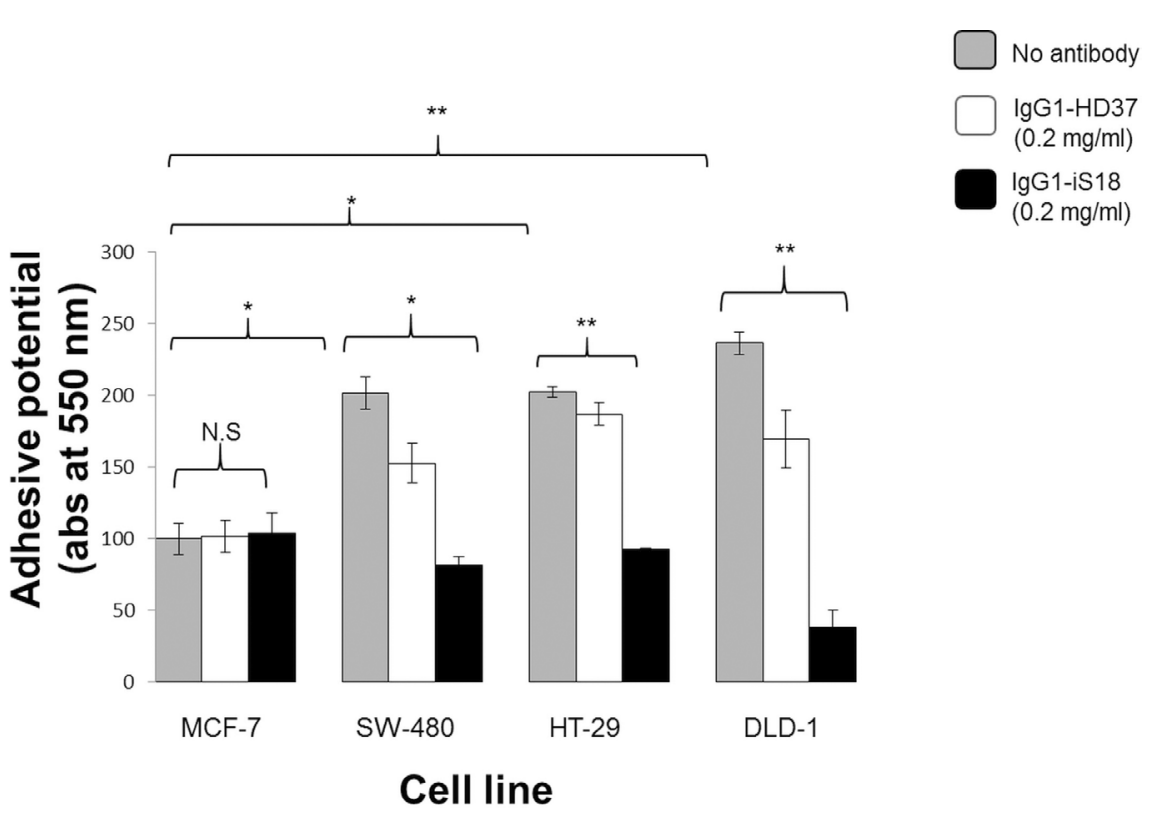

Figure 5. Adhesion of tumorigenic cells to laminin- 1 investigated through adhesion assays. Each cell line's untreated cells were compared with MCF-7 untreated control cells to determine adhesive potential. $P$ values show all three cell lines had increased adhesive potential compared to MCF-7. After treatment with the anti-LRP/LR-specific antibody IgG1-iS18, $P$ values showed significantly decreased adhesive potential compared to MCF-7 control cells; SW- 480 by $59 \%$, HT- 29 by $56 \%$ and DLD- 1 by $84 \%$. The control antibody IgG1-HD37 shows a small effect on each colorectal cancer cell line; however, statistical analysis indicates that IgG1-iS18 has a significantly greater effect $\left({ }^{* * *} p=0.001106\right.$ in SW-480, ${ }^{* * *} P=2.97188 \mathrm{E}-05$ for HT-29 and ${ }^{* * *} p=0.000644$ in DLD-1).

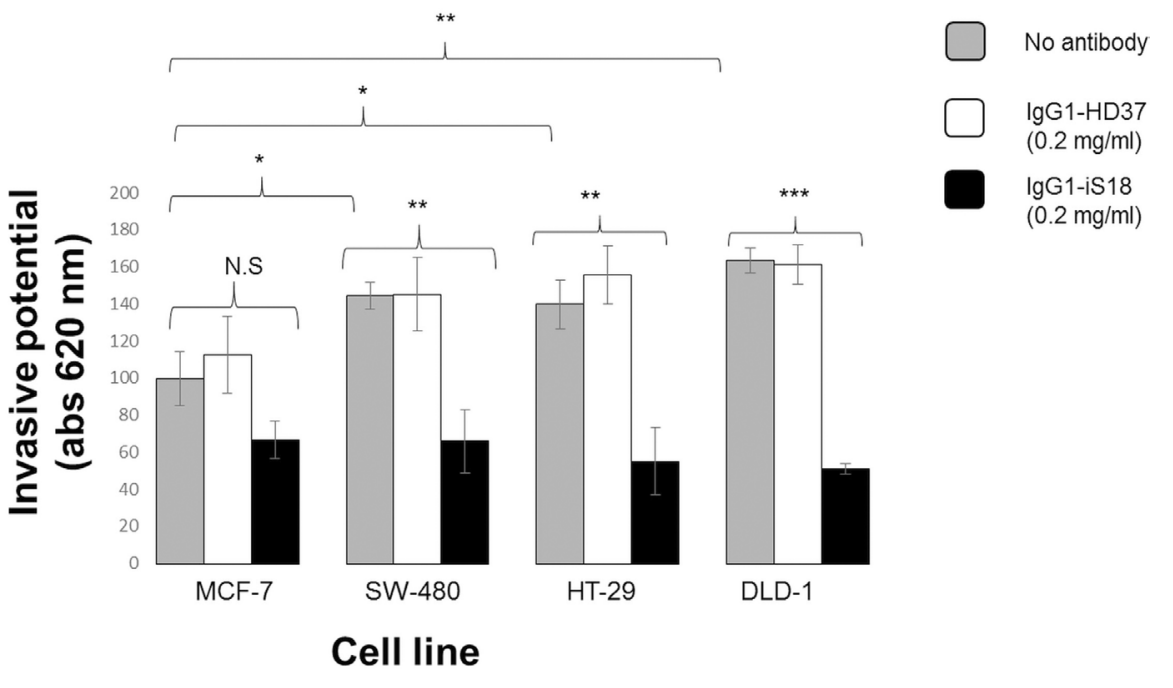

Figure 6. Invasion of ECM-like Matrigel ${ }^{\mathrm{TM}}$ by tumorigenic cell lines. Each cell line's untreated cells were compared with MCF-7 untreated control cells to determine invasive potential. $P$ values show that all three colorectal cancer cell lines had an increase in invasive potential compared to MCF-7. After treatment with the anti-LRP/LR-specific antibody, P values showed significantly decreased adhesive potential compared to MCF-7 control cells: SW480 by $54 \%$, HT-29 by $60 \%$ and DLD-1 by $69 \%$. The control antibody lgG $1-H D 37$ did not affect the invasive potential of the tumorigenic cell lines as expected. invasive, there is no difference when the colorectal cancer cell lines are compared with one another. However, Figure 4 shows that with total LRP/LR levels, there is a significant two-fold increase between early-stage (SW-480 and HT29) and late-stage (DLD-1) colorectal cancer cells. Hence, the lower total LRP / LR levels observed for SW-480 and HT-29 (Figure 5) could account for the lower adhesive and invasive potential for these cells lines, while high levels of total LRP/LR leads to the higher adhesive and invasive potential of DLD-1 (Figure 6). This could also account for IgG1-iS18 being most effective in latestage colorectal cancer, since this stage was observed to contain the highest levels of LRP/LR, which the antibody is targeted against. Evaluation of the correlation between cell surface and total LRP/LR levels and the adhesive and invasive potential of early- and late-stage colorectal cancer cells showed a substantially high correlation (Tables 2 and 3). This indicates a relationship between the two factors that is positive and directly proportional. The high correlation coefficients found for total and cell surface LRP/LR levels to the adhesive and invasive potential of each cell line suggests that cancer progression or aggressiveness is enhanced by increased levels of LRP/LR, which correlates with previous studies performed by Omar et al. (30).

The significant reduction in invasive potential of both stages of colorectal cancer upon treatment with IgG1-iS18 may be a result of inhibiting adhesion (Figure 5), since adhesion is known to be a prerequisite for invasion to occur, and both steps are needed to induce metastasis. This was affirmed by the high Pearson's correlation coefficients between the adhesive and invasive potential for all three cell lines (Tables 2 and 3). These results coincide with previous studies showing that the interaction between LRP/LR and laminin-1 is necessary for adhesion as well as secretion of important enzymes needed to degrade the basement membrane, consequently promoting invasion (4). 
Table 1. Median fluorescence intensity (MFI) values used as an indicator of differential expression of LRP/LR on the surface of MCF-7, SW-480, HT-29 and DLD-1 cells.

\begin{tabular}{lccc}
\hline Cell line & $\begin{array}{c}\text { MFI of unlabelled } \\
\text { cells (A) }\end{array}$ & $\begin{array}{c}\text { MFI of cells stained with } \\
\text { lgG1-iS18 and PE-coupled } \\
\text { secondary antibody (B) }\end{array}$ & $\begin{array}{c}\text { Difference in MFI } \\
\text { values : (B) - (A) }\end{array}$ \\
\hline MCF-7 & 3768.83 & 7308.67 & 3539.84 \\
SW-480 & 3592.67 & 24244 & 20651.33 \\
HT-29 & 11894.17 & 32351.83 & 20457.67 \\
DLD-1 & 6713.67 & 28045.33 & 21331.67 \\
\hline
\end{tabular}

All MFI values represent an average of experiments performed in triplicate.

Table 2. Pearson's correlation coefficients between cell surface LRP/LR levels and the adhesive and invasive potential of early-stage (SW-480 and HT-29) and late-stage (DLD-1) colorectal cancer cells.

\begin{tabular}{lccc}
\hline Cell line & $\begin{array}{c}\text { LRP/LR level to adhesive } \\
\text { potential }\end{array}$ & $\begin{array}{c}\text { LRP/LR level to invasive } \\
\text { potential }\end{array}$ & $\begin{array}{c}\text { Adhesive to invasive } \\
\text { potential }\end{array}$ \\
\hline SW-480 & 0.99 & 0.99 & 0.91 \\
HT-29 & 0.96 & 0.85 & 0.98 \\
DLD-1 & 0.99 & 0.89 & 0.94 \\
\hline
\end{tabular}

Table 3. Pearson's correlation coefficients between total LRP/LR levels and the adhesive and invasive potential of early-stage (SW-480 and HT-29) and late-stage (DLD-1) colorectal cancer cells.

\begin{tabular}{lccc}
\hline Cell line & $\begin{array}{c}\text { LRP/LR level to adhesive } \\
\text { potential }\end{array}$ & $\begin{array}{c}\text { LRP/LR level to invasive } \\
\text { potential }\end{array}$ & $\begin{array}{c}\text { Adhesive to invasive } \\
\text { potential }\end{array}$ \\
\hline SW-480 & 0.99 & 0.98 & 0.91 \\
HT-29 & 0.98 & 0.99 & 0.98 \\
DLD-1 & 0.98 & 0.94 & 0.94 \\
\hline
\end{tabular}

Numerous studies have also investigated the pathological potential of the LRP/LR-laminin-1 interaction through the use of various anti-LRP/ LR tools such as monoclonal antibodies (26), transdominant negative mutants (27) and pentosan sulphates (5). These strategies all show significant decreases in the adhesive and invasive potential of particular carcinomas through blockage of the LRP/LR-laminin-1 interaction. Along with these findings, it is proposed that this interaction significantly promotes proteolytic cleavage of the basement membrane and consequently aids in invasion and metastasis. According to the findings of this study, the anti-LRP/LR-specific antibody IgG1-iS18 is able to significantly impede critical steps of metastasis, adhesion and invasion of early- and late-stage colorectal cancer in vitro. Therefore, this suggests IgG1-iS18 as a promising therapeutic tool in treating early- and late-stage metastatic colorectal cancer. Finally, to deem this antibody as a therapeutic tool against cancer, further in vivo analysis must be performed with animal studies. Once this is achieved, further conclusions can be made on whether IgG1-iS18 has the potential to be a therapeutic tool for the treatment of metastatic colorectal cancer.

\section{CONCLUSION}

The anti-LRP/LR-specific antibody IgG1-iS18 was shown to have a significant impact on the behavior of SW-480, HT-29 and DLD-1 cancer cells by impeding their adhesive and invasive potential in vitro, suggesting that this antibody may act as a possible therapeutic tool for the treatment of metastatic colorectal cancer.

\section{ACKNOWLEDGEMENTS}

This work was supported by the National Research Foundation, Republic of South Africa. Any opinions, findings and conclusions, or recommendations expressed in this material are those of the authors, and the National Research Foundation does not accept any liability in this regard. The funders had no role in study design, data collection and analysis, decision to publish, or preparation of the manuscript. The research from which this publication emanated was co-funded by the South African Medical Research Council. We also thank Affimed $\mathrm{GmbH}$, Heidelberg, Germany for providing the antibody IgG1-iS18.

\section{DISCLOSURE}

The authors declare that they have no competing interests as defined by Molecular Medicine, or other interests that might be perceived to influence the results and discussion reported in this paper.

\section{REFERENCES}

1. World Health Organization. (2012) Globocan 2012: Estimating Cancer Incidence, Mortality and Prevalence Worldwide.

2. Bray F, Jemal A, Grey N, Ferlay J, Forman M. (2012) Global cancer transitions according to the Human Development Index (2008-2030): a population-based study. Lancet Oncol. 13(8):790-801.

3. Kushi LH, Doyle C, McCullough M. (2012) American Cancer Society guidelines on nutrition and physical activity for cancer prevention: reducing the risk of cancer with healthy food choices and physical activity. CA Cancer J. Clin. 62(1):30-67.

4. Hanahan D, Weinberg RA. (2000)The Hallmarks of Cancer. Cell. 100:57-70

5. Fülöp T, Larbi A. (2002) Putative role of $67 \mathrm{kDa}$ elastin-laminin receptor in tumour invasion. Sem. Cancer Biol. 12(3):219-229.

6. Zuber C, et al. (2008) Invasion of tumourigenic HT1080 cells is impeded by blocking or downregulating the $37-\mathrm{kDa} / 67-\mathrm{kDa}$ laminin receptor. J. Mol. Biol. 378(3): 530-39.

7. Rao CN, Castrovono V, Schmitt MC. (1989) Evidence for a precursor of the high-affinity 
metastasis-associated murine laminin receptor. Biochemistry. 28:7476-86.

8. Chetty C, et al. (2014). Anti-LRP/LR specific antibody IgG1-iS18 impedes adhesion and invasion of liver cancer cells. PLoS ONE. 9(5):e96268.

9. Sato M, et al. (1996) Analysis of nuclear localization of laminin binding protein precursor p40 (LBP/p40). Biochem. Biophys. Res. Commun. 229:896-901.

10. Kinoshita K, et al. (1998) LBP-p40 binds DNA tightly through associations with histones H2A, $\mathrm{H} 2 \mathrm{~B}$, and H4. Biochem. Biophys. Res. Commun. 253(2):277-82.

11. DiGiacomo V, Meruelo D. (2016) Looking into laminin receptor: non-integrin 37/67-kDa laminin receptor/RPSA protein. Biol. Rev. Camb. Philos. Soc. 91(2):288-310.

12. Khusal R, et al. (2013) In vitro inhibition of angiogenesis by antibodies directed against the 37-kDa/67-kDa laminin receptor. PLoS ONE. 8(3):e58888.

13. Rieger R, et al. (1997) The human $37 \mathrm{kDa}$ laminin receptor precursor interacts with prion protein in eukaryotic cells. Nat. Med. 3(12):1383-88.

14. Gauczynski S, et al. (2001) The $237 \mathrm{kDa} / 67$ $\mathrm{kDa}$ laminin receptor acts as the cell surface receptor for the cellular prion protein. EMBO J. 20(21):5863-75.

15. Hundt C, et al. (2001) Identification of interaction domains of the prion protein with its $37 \mathrm{kDa} / 67$ kDa laminin receptor. EMBO J. 20(21):5876-86.

16. Jovanovic K, et al. (2013) Anti-LRP/LR specific antibodies and shRNAs impede amyloid beta shedding in Alzheimer's disease. Sci. Rep. 3:2699.

17. Da Costa Dias, et al. (2013) Anti-LRP/LR specific antibody IgG1-iS18 and knock-down of LRP/LR by shRNAs rescue cells from Abeta42 induced cytotoxicity. Sci. Rep. 3:2702.

18. Jovanovic K, et al. (2014) High resolution imaging study of interactions between the $37 \mathrm{kDa} / 67 \mathrm{kDa}$ laminin receptor and APP, beta-secretase and gamma-secretase in Alzheimer's disease. PLOS One. 9(6):e100373.

19. Da Costa Dias B, et al. (2014) The $37 \mathrm{kDa} / 67 \mathrm{kDa}$ laminin receptor acts as a receptor for $\mathrm{A} \beta_{42}$ internalization. Sci. Rep. 4:5556.

20. Pinnock EC, et al. (2015) LRP/LR antibody mediated rescuing of Abeta induced cytotoxicity is dependent on PrPc in Alzheimer's disease. J. Alzheimers Dis. 43:3.

21. Naidoo K, et al. (2015) Knock-down of the $37 \mathrm{kDa} / 67 \mathrm{kDa}$ laminin receptor LRP/LR impedes telomerase activity. PLoS One. 10:11.

22. Nelson J, et al. (2008) The $67 \mathrm{kDa}$ laminin receptor: structure, function and role in disease. Biosci Rep. 28:33-48.

23. Omar A, et al. (2011) Patented biological approaches for the therapeutic modulation of the $37 \mathrm{kDa} / 67 \mathrm{kDa}$ laminin receptor. Expert Opin. Ther. Pat. 21:35-53.

24. Sasaki T, Fassler R, Hohenester E. (2004) Laminin: the crux of the basement membrane assembly. J. Cell Biol. 164(7):959-63.
25. Ryan MC, et al. (1996) The functions of laminins: lessons from in vivo studies. Matrix Biol. 15:369-81.

26. Weeks BS, DiSalvo J, Kleinman HK. (1990) Laminin-mediated process formation in neuronal cells involves protein dephosphorylation. J. Neurosci. Res. 27:418-26.

27. Givant-Horwitz V, Davidson B, Reich R. (2004) Laminin-induced signaling in tumour cells: the role of the M (r) 67,000 laminin receptor. Cancer Res. 64:3572-79.

28. Jovanovic K, et al. (2015) Novel patented therapeutic approaches targeting the $37 / 67 \mathrm{kDa}$ laminin receptor for treatment of cancer and Alzheimer's disease. Expert Opin. Ther. Patents. 25(5):1-5.

29. Pelosi G, et al. (1997) High-affinity monomeric 67-kD laminin receptors and prognosis in pancreatic endocrine tumours. J. Pathol.183:62-69.

30. Omar A, et al. (2012) Anti-LRP/LR-specific antibody IgG1-iS18 significantly reduces adhesion and invasion of metastatic lung, cervix, colon and prostate cancer cells. J. Mol. Biol. 419:102-09.

31. Khumalo T, et al. (2013) Adhesion and invasion of breast and oesphageal cancer cells is impeded by anti-LRP/LR-specific antibody IgG1-iS18. PLOS ONE. 8(6):e66297.

Cite this article as: Vania L, Chetty CJ, Ferreira E, Weiss SFT. (2016) Anti-LRP/LR-Specific antibody IgG1-iS18 significantly impedes adhesion and invasion in early- and late-stage colorectal carcinoma cells. Mol. Med. 22:664-73. 